\section{Recurrent acute pancreatitis secondary to pancreatic neuroendocrine tumor: role of endoscopic ultrasound}

\section{Introduction}

Recurrent acute pancreatitis due to a neuroendocrine tumor (NET) is very rare. Endoscopic ultrasound (EUS) provides excellent anatomic details of the pancreas. We present two cases of recurrent acute pancreatitis due to NET. EUS picked up small space occupying lesions in both the cases which were not picked up by CT scan and were later confirmed to be NET by fine needle aspiration cytology (FNAC).

\section{Case reports}

Case 1

A 34-year-old female had four episodes of acute pancreatitis over last one year as diagnosed by characteristic abdominal pain, raised serum amylase and serum lipase levels and ultrasound abdomen. Her history and biochemical investigations could not delineate the etiology of pancreatitis. A contrast enhanced CT of abdomen was performedwhich showed pancreatic duct dilation upto the neck of pancreas.However the cause of obstruction at the neck could not be ascertained. EUS was carried out and it showed a homogenous, hypoechoic space occupying lesion at the neck of pancreas,measuring $8 \mathrm{~mm}$ in size and obstructingthe pancreatic duct (Figure 1) which measured $3 \mathrm{~mm}$. An FNAC from this lesion showed the picture of NET. Patient could not undergo surgery because she became pregnant and is under follow-up since lastfour months.

\section{Case 2}

A 40-year-old female presented with three episodes of acute pancreatitis diagnosed by characteristic abdominal pain, raised serum amylase and lipase levels during episodes of pain and ultrasound abdomen suggestive of acute pancreatitis sincetwo years. Her biochemical investigations and MRI abdomen with MRCP and contrast enhanced CT abdomen did not reveal the etiology of acute pancreatitis. EUS was performed and it showed a $1 \mathrm{~cm}$ lesion at the pancreatic neck along with a dilated

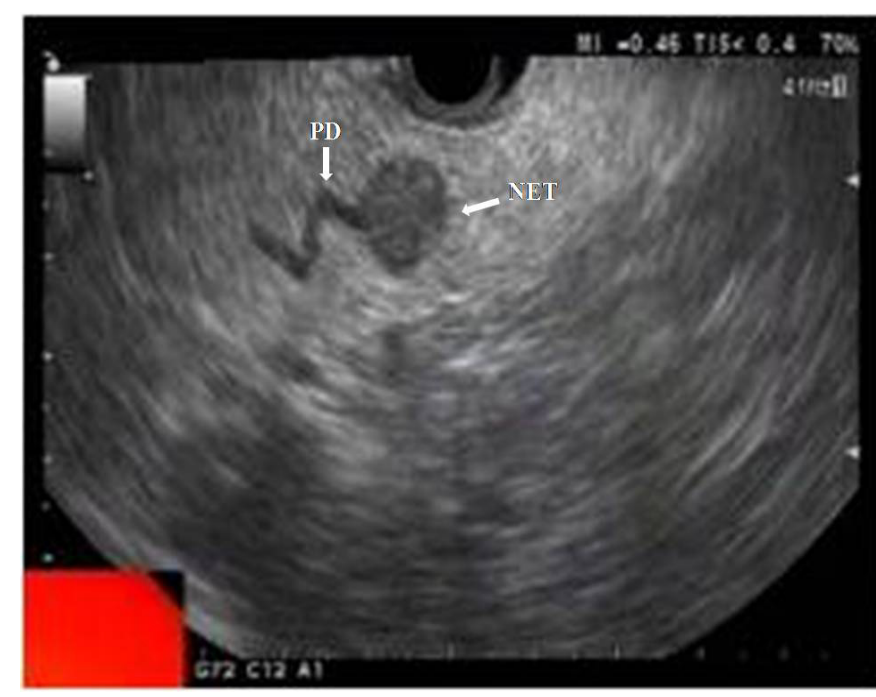

Figure 1: $\quad$ EUS image showing space occupying lesion at neck of pancreas and dilated main pancreatic duct

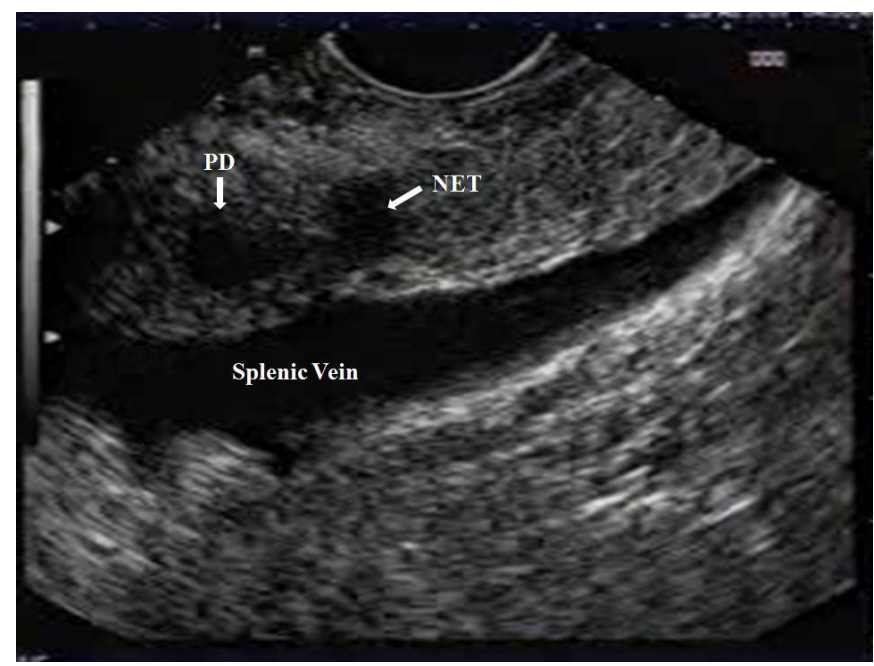

Figure 2: $1 \mathrm{~cm}$ lesion at the pancreatic neck along with a dilated pancreatic duct

pancreatic duct (Figure 2). An FNAC from this lesion was suggestive of NET. The patient underwent resection of the pancreatic tumor. She is under follow-up and is asymptomatic since four years.

\section{Discussion}

It is important to determine the etiology of recurrent acute pancreatitis in order to prevent recurrent attacks and complications. NET are rare tumors of the pancreas. They may secrete hormones or may be non-functional, presenting due to secondary mass effect. ${ }^{1}$ EUS offers the advantage of excellent anatomic details of the pancreas as compared to CT. On EUS, NET generally appears hypoechoic, homogeneous and well defined although they may be isoechoic or hyperechoic as well, with irregular margins. ${ }^{2}$ EUS has a sensitivity, specificity 
and diagnostic accuracy approaching 90\% for the diagnosis of pancreatic NET and performs better than CT. ${ }^{3-5} \mathrm{EUS}$ also facilitates superior FNAC sampling. Overall sensitivity of EUSguided FNAC approaches $90 \% .{ }^{6}$ NET rarely presents as acute pancreatitis..$^{7-9}$ Our case reports suggest that EUS is important step in the diagnostic work-up of patients with undetermined etiology of recurrent acute pancreatitis. To conclude we report two cases of recurrent acute pancreatitis due to NET diagnosed by EUS, which were not detected by conventional imaging modalities.

RAJESH PURI

NARENDRA S CHOUDHARY

RANDHIR SUD

Correspondence: Dr. Rajesh Puri Institute of Digestive and Hepatobiliary Sciences, Medanta, The Medicity, Gurgaon, India

Email:purirajesh69@gmail.com

\section{References}

1. Figueiredo FA, Giovannini M, Monges G, Charfi S, Bories E, Pesenti C, et al. Pancreatic endocrine tumors: a large single-center experience. Pancreas. 2009;38:936-40.

2. Lee LS. Diagnosis of pancreatic neuroendocrine tumors and the role of endoscopic ultrasound. Gastroenterol Hepatol (N Y). 2010;6:520-2.

3. Pais SA, Al-Haddad M, Mohamadnejad M, Leblanc JK, Sherman S, McHenry L, et al. EUS for pancreatic neuroendocrine tumors: a single-center, 11-year experience. Gastrointest Endosc. 2010;71:1185-93.

4. Anderson MA, Carpenter S, Thompson NW, Nostrant TT, Elta GH,Scheiman JM. Endoscopic ultrasound is highly accurate and directs management in patients with neuroendocrine tumors of the pancreas. Am J Gastroenterol. 2000;95:2271-7.

5. Gouya H, Vignaux O, Augui J, Dousset B, Palazzo L, Louvel A, et al. CT, endoscopic sonography, and a combined protocol for preoperative evaluation of pancreatic insulinomas. AJR Am J Roentgenol. 2003;181:987-92.

6. Figueiredo FA, Giovannini M, Monges G, Bories E, Pesenti C, Caillol F, et al. EUS-FNA predicts 5-year survival in pancreatic endocrine tumors. Gastrointest Endosc. 2009;70:907-14.

7. Grino P, Martinez J, Grino E, Carnicer F, Alonso S, PerezBerenguer $\mathrm{H}$, et al. Acute pancreatitis secondary to pancreatic neuroendocrine tumours. JOP. 2003;4:104-10.

8. Tejedor Bravo M, Justo LM, Lasala JP, Moreira Vicente VF, Ruiz AC, Scapa Mde L. Acute pancreatitis secondary to neuroendocrine pancreatic tumors: report of 3 cases and literature review. Pancreas. 2012;41:485-9.

9. Jukemura J, Montagnini AL, Perini MV, de Almeida JL, Rodrigues JJ, da Cunha JE. Acute pancreatitis associated with neuroendocrine tumor of the pancreas. JOP. 2006;7:56-61. 